\section{ORIGINAL RESEARCH}

M. Wintermark

S.S. Jawadi

J.H. Rapp

T. Tihan

E. Tong

D.V. Glidden

S. Abedin

S. Schaeffer

G. Acevedo-Bolton

B. Boudignon

B. Orwoll

X. Pan

D. Saloner

\title{
High-Resolution CT Imaging of Carotid Artery Atherosclerotic Plaques
}

BACKGROUND AND PURPOSE: Plaque morphologic features have been suggested as a complement to luminal narrowing measurements for assessing the risk of stroke associated with carotid atherosclerotic disease, giving rise to the concept of "vulnerable plaque." The purpose of this study was to evaluate the ability of multidetector-row CT angiography (CTA) to assess the composition and characteristics of carotid artery atherosclerotic plaques with use of histologic examination as the gold standard.

MATERIALS AND METHODS: Eight patients with transient ischemic attacks who underwent carotid CTA and "en bloc" endarterectomy were enrolled in a prospective study. An ex vivo micro-CT study of each endarterectomy specimen was obtained, followed by histologic examination. A systematic comparison of CTA images with histologic sections and micro-CT images was performed to determine the CT attenuation associated with each component of the atherosclerotic plaques. A computer algorithm was subsequently developed that automatically identifies the components of the carotid atherosclerotic plaques, based on the density of each pixel. A neuroradiologist's reading of this computer analysis was compared with the interpretation of the histologic slides by a pathologist with respect to the types and characteristics of the carotid plaques.

RESULTS: There was a $72.6 \%$ agreement between CTA and histologic examination in carotid plaque characterization. CTA showed perfect concordance for calcifications. A significant overlap between densities associated with lipid-rich necrotic core, connective tissue, and hemorrhage limited the reliability of individual pixel readings to identify these components. However, CTA showed good correlation with histologic examination for large lipid cores $(\kappa=0.796 ; P<.001)$ and large hemorrhages $(\kappa=0.712 ; P=.102)$. CTA performed well in detecting ulcerations $(\kappa=0.855)$ and in measuring the fibrous cap thickness $\left(R^{2}=0.77 ; P<.001\right)$.

CONCLUSION: The composition of carotid atherosclerotic plaques determined by CTA reflects plaque composition defined by histologic examination.

$\mathbf{L}$ uminal narrowing is the standard parameter used in reporting the extent and severity of carotid artery stenosis. The widespread use of this measure is based primarily on the results of several randomized clinical trials that demonstrated a reduction in the risk for ischemic stroke in patients with luminal stenosis of $70 \%$ or greater (assessed on conventional angiograms), after carotid endarterectomy compared with medical treatment alone. ${ }^{1-4}$ However, carotid stenosis of $70 \%$ or more occurs in less than $10 \%$ of patients, whereas less than $70 \%$ of carotid stenosis is extremely frequent in the general population $(70 \%$ in men and $60 \%$ in women 64 years of age). ${ }^{5,6}$ In patients with less than $70 \%$ carotid stenosis, highresolution lumenography fails to provide any insight into the

Received August 2, 2007; accepted after revision November 29

From the Departments of Radiology, Neuroradiology Section (M.W., E.T., S.S., G.A.-B., D.S.); Pathology (T.T.); Epidemiology and Biostatistics (D.V.G.); and Division of Endocrinology (B.B., B.O.), University of California, San Francisco, Calif.; Department of Vascular Surgery (J.H.R., X.P.), San Francisco Veterans Administration Medical Center, San Francisco, Calif; and Department of Diagnostic Radiology (S.A., S.S.J.), University of Missouri-Kansas City School of Medicine, Kansas City, Mo.

This work was supported by a Fellowship in Basic Science Research from the Berlex/ Neuroradiology Education \& Research Foundation, by a VA MERIT review award, and by Grant KL2 RR024130 from the National Center for Research Resources (NCRR), a component of the National Institutes of Health $(\mathrm{NIH})$ and the NIH Roadmap for Medical Research. Its contents are solely the responsibility of the authors and do not necessarily represent the official view of NCRR or NIH. Information on NCRR is available at http://www.ncrr.nih.gov/. Information on Re-engineering the Clinical Research Enterprise can be obtained from http://nihroadmap.nih.gov/clinicalresearch/overview-translational.asp.

Please address correspondence to Max Wintermark, MD, Department of Radiology, Neuroradiology Section, University of California San Francisco, 505 Parnassus Ave, Box 0628, San Francisco, CA 94143-0628; e-mail: max.wintermark@radiology.ucsf.edu DOI 10.3174/ajnr.A0950 associated risk for stroke, because angiography is able to detect atherosclerosis only when more than $40 \%$ of the area of the vessel wall is occupied by the plaque. ${ }^{7}$

Plaque morphologic features and composition have been suggested as a complement to luminal narrowing measurements for assessing carotid atherosclerotic disease, giving rise to the concept of "vulnerable plaque." ${ }^{-12}$ Several carotid morphologic features have been reported as being associated with an increased risk for stroke, the most studied descriptor being the common carotid artery (CCA) intima-media thickness. ${ }^{5,6,13-17}$ Carotid plaques with a thin fibrous cap and a large lipid core are also considered to increase the risk for stroke, ${ }^{18,19}$ as are ulcerated plaques. ${ }^{20}$ In contrast, plaques with high calcium content, especially when located superficially, are thought to be associated with a lower risk for stroke. ${ }^{21}$

Noninvasive in vivo imaging of carotid atherosclerotic plaques holds considerable promise for clinical decision making and treatment. ${ }^{18,22,23}$ Such imaging has classically been achieved with sonography ${ }^{5,6,13-17}$ and MR imaging. ${ }^{3,24-29}$ It is surprising that only a few studies have evaluated carotid wall descriptors with $\mathrm{CT},{ }^{30-32}$ though CT angiography (CTA) is a well-established technique frequently used to assess carotid stenosis. ${ }^{30,33,34}$ Previous studies that explored CTA as a means of imaging atherosclerotic plaques have involved older-generation, single-section CT scanners ${ }^{31,32,35-40}$ and have usually focused on 1 single component, such as calcium. ${ }^{36,38,39,41}$

The goal of this study was to evaluate the ability of modern, multidetector-row, isotropic resolution CTA studies to assess 


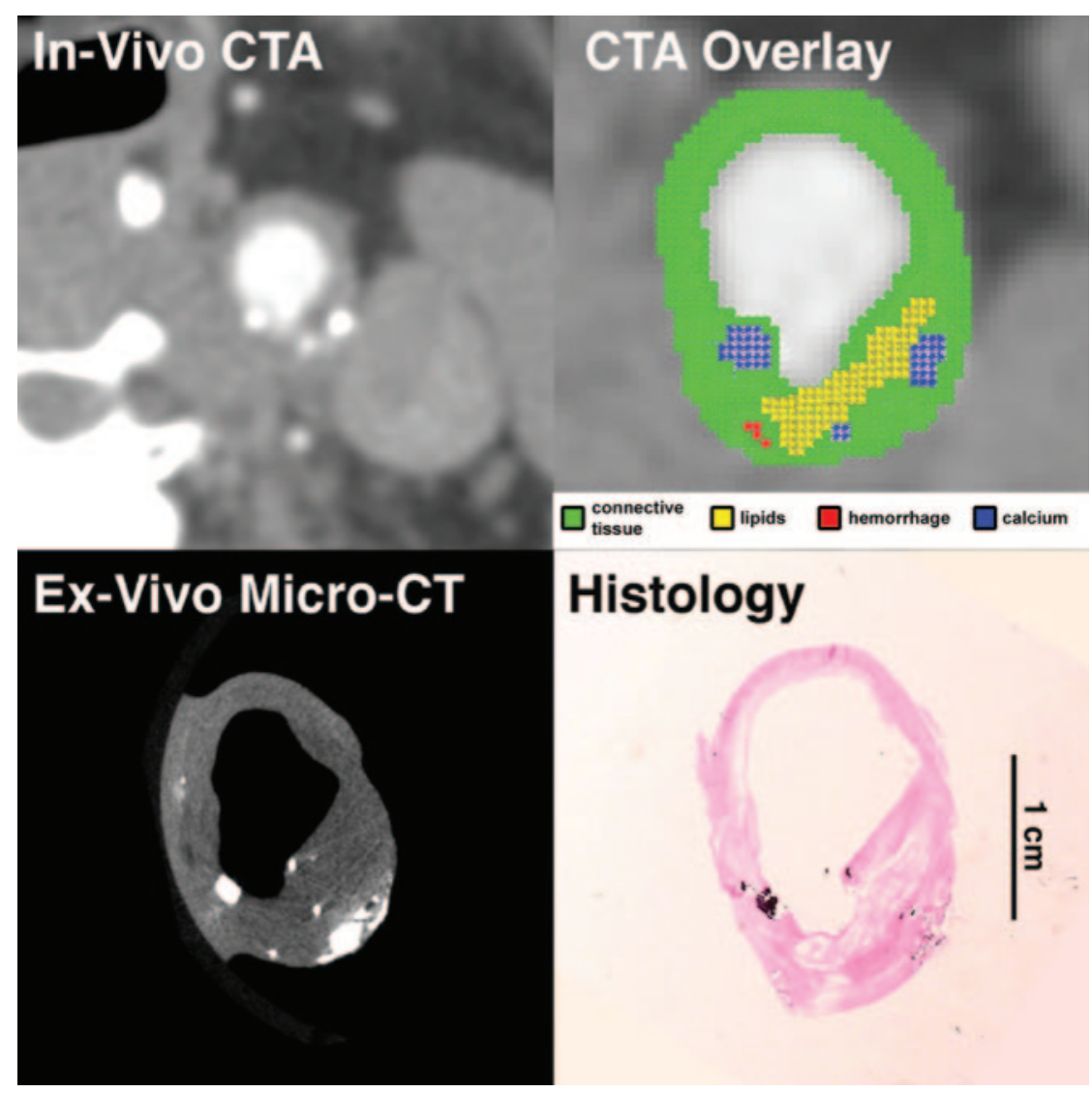

Fig 1. In vivo CTA image of the common carotid artery, and matching ex vivo micro-CT and histologic sections. Automated classification computer algorithm-derived overlay shows lipid-rich necrotic core (yellow), calcification (blue), blood products (red), and remaining connective tissue (green) CTA overlay demonstrates a plaque with a large lipid core, small calcifications, and an ulceration, making it a Vla lesion according to the AHA classification, in agreement with histologic examination, the gold standard for noncalcified carotid wall components, and with ex vivo micro-CT, the reference for carotid wall calcium (specimens were decalcified before histologic sectioning).

$0.625 \mathrm{~mm}$; pitch, 1.375:1; section thickness, $0.625 \mathrm{~mm}$; reconstruction interval, $0.5 \mathrm{~mm}$; and acquisition parameters: $120 \mathrm{kVp} / 240 \mathrm{~mA}$. A caudocranial scanning direction was selected, covering from the midchest to the vertex. Iohexol (Omnipaque; Amersham Health, Princeton, NJ; $300 \mathrm{mg} / \mathrm{mL}$ of iodine) $70 \mathrm{~mL}$ was injected to an antecubital vein with a power injector at a rate of $4 \mathrm{~mL} / \mathrm{s}$. Optimal timing of the CTA acquisition was achieved with use of a test bolus technique.

\section{Ex Vivo Micro-CT Imaging Protocol of} the Carotid Endarterectomy Specimens The carotid endarterectomy specimens were imaged with a VivaCT 40 micro-CT scanner (Scanco Medical, Southeastern, $\mathrm{Pa}$ ) with the following parameters: $70 \mathrm{kVp}, 160 \mu \mathrm{A}, 30-\mu \mathrm{m}$

the histologic composition (including noncalcified components) and characteristics of carotid artery atherosclerotic plaques with use of histologic examination as the gold standard.

\section{Methods}

\section{Study Design}

Eight patients with transient ischemic attacks (TIA) underwent a CTA study, were found to have more than $50 \%$ carotid stenosis, and were scheduled for carotid endarterectomy as part of their standard of care. They were enrolled in a prospective study approved by our institutional review board. As part of the research protocol, patients were asked to provide permission for their preoperative CTA study and endarterectomy specimen to be used for research purposes. The endarterectomy specimens were excised en bloc according to a technique described previously in the literature. ${ }^{26}$ An ex vivo micro-CT study of each specimen was obtained, followed by ex vivo histologic examination. Two analyses, 1 quantitative and the other qualitative, were performed, comparing in vivo CTA to histologic examination, the gold standard for noncalcified carotid wall components, and to ex vivo micro-CT, the reference for carotid wall calcium (specimens were decalcified before histologic sectioning). Details of each analysis are described below and are derived from the methodology recommended by Lovett et $\mathrm{al}^{42}$ for comparing carotid plaque imaging to histologic features.

\section{In Vivo CTA Imaging Protocol}

The CTA studies were obtained on a 16-section CT scanner (GE Healthcare, Milwaukee, Wis). The image acquisition protocol was as follows: spiral mode, 0.6 -second gantry rotation; collimation, $16 \times$ section thickness, and $30-\mu \mathrm{m}$ isotropic resolution.

\section{Ex Vivo Histologic Processing of the Carotid Endarterectomy Specimens}

After micro-CT imaging, plaques were decalcified and sectioned transverse to the lumen. Depending on the length of the specimen, histologic sections were performed at 4 to 9 locations, at the level of the bifurcation and every $3 \mathrm{~mm}$ both proximal and distal to the bifurcation. These locations were marked in the common carotid artery (CCA) at $0 \mathrm{~mm},-3 \mathrm{~mm},-6 \mathrm{~mm},-9 \mathrm{~mm},-12 \mathrm{~mm}$, and $-15 \mathrm{~mm}$, and in the internal carotid artery (ICA) at $3 \mathrm{~mm}, 6 \mathrm{~mm}, 9 \mathrm{~mm}, 12 \mathrm{~mm}$, and $15 \mathrm{~mm}$. The resulting blocks were embedded in paraffin and sectioned in a microtome. Sections were stained with hematoxylineosin and Oil Red O to identify connective tissue, lipid-rich necrotic core, and blood products. Transparent stereologic grid cover slips (Bellco Biotechnology, Vineland, NJ) were mounted onto the slides to allow the characterization of the carotid wall in distinct $2 \times 2-\mathrm{mm}$ squares. Digital images of the histologic preparations were acquired at a $25-\mu \mathrm{m}$ in-plane resolution.

\section{Postprocessing of In Vivo CTA Images and Ex Vivo Micro-CT Images}

The in vivo CTA images and ex vivo micro-CT images were registered to the histologic slides by linear registration (scaling, translation, and rotation transformation only) with the ImageJ plug-in Align 3TP. The scaling factor was known from the relative size of the CTA and micro-CT pixels. Translation and rotation were used to obtain reformatted images matching the orientation of the corresponding histologic sections. CT sections were matched by observing the overall morphologic features of the plaque and incorporating the known location and distance of the section from the carotid bifurcation, according to a 


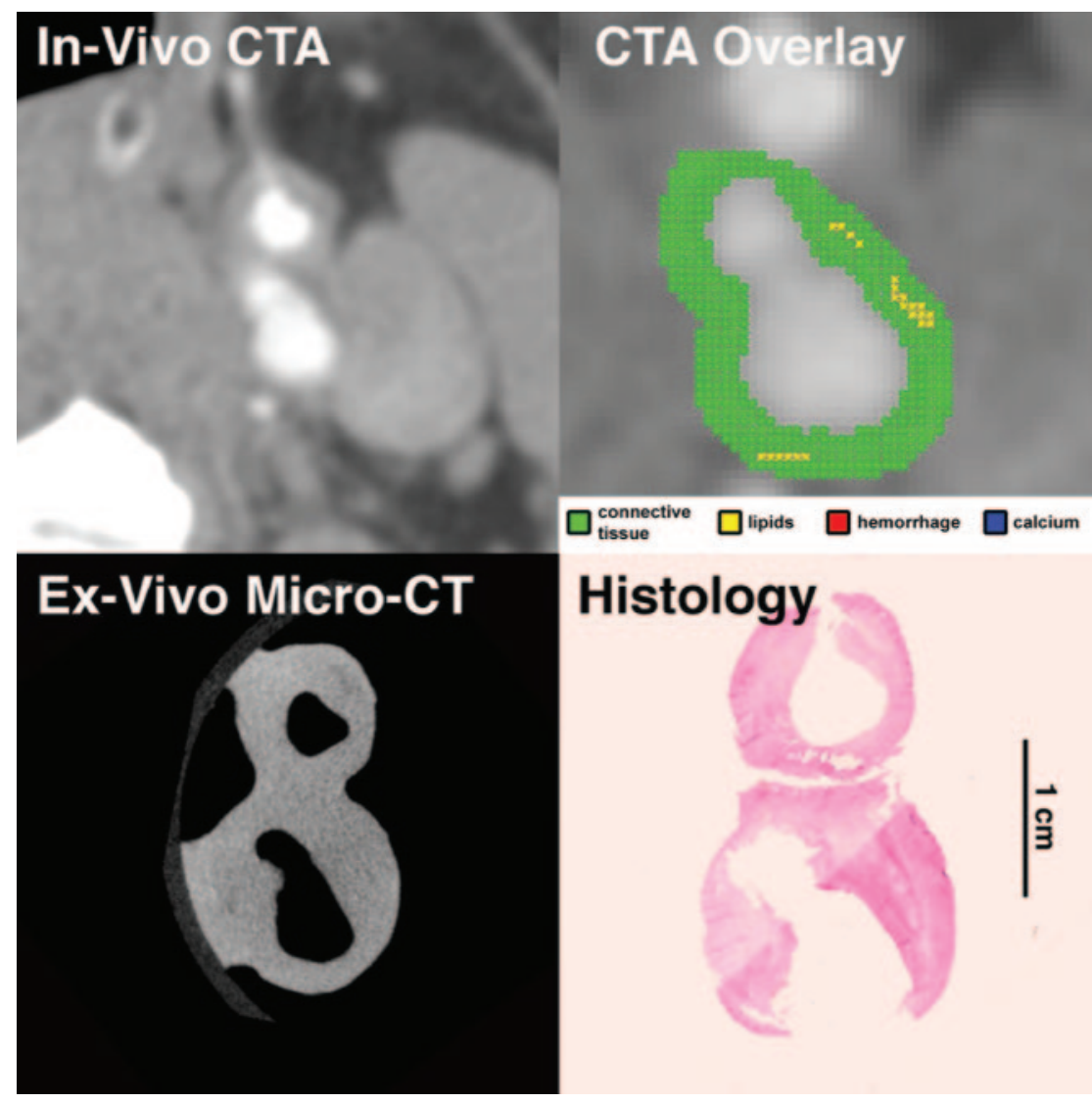

Fig 2. In vivo CTA image of the ICA, and matching ex vivo micro-CT and histologic sections. Automated classification computer algorithm-derived overlay demonstrates a plaque with sparse "lipid" pixels (yellow) and an ulceration, making it a Vla lesion according to the AHA classification, in agreement with histologic examination.

lipid-rich necrotic core, hemorrhage, and calcifications in each of the $2 \times 2-\mathrm{mm}$ squares were calculated.

The in vivo CTA reformatted images were reviewed independent of the histologic slides. The average CT Hounsfield attenuation was recorded in each of the $2 \times 2$-mm squares electronically drawn on the reformatted images in the same orientation and location as on the corresponding histologic slides.

Using a linear mixed model of the average CT Hounsfield attenuation in each of the $2 \times$ 2-mm squares (outcome) with respect to the percentages of connective tissue, lipid-rich necrotic core, hemorrhage, and calcifications in the corresponding histologic squares (predictors), with a random effect for patient, and assuming the errors were normal, we determined the mean Hounsfield attenuation for each histologic component (connective tissue, lipidrich necrotic core, hemorrhage, and calcifications), as well as the $95 \%$ confidence intervals for these densities. This linear mixed model

method described previously. ${ }^{25}$ Grids of $2 \times 2$-mm squares were electronically drawn on the reformatted images in the same orientation and location as on the corresponding histologic slides. To avoid any bias, the process of orienting and localizing CTA and micro-CT images with histologic examination was performed by a separate reviewer, before and independent of the qualitative and quantitative analyses described below.

\section{Quantitative Analysis}

A pathologist, who was blinded to the CTA images, independently reviewed the histologic slides in combination with the matching micro-CT images and evaluated the composition of the carotid walls in each $2 \times 2-\mathrm{mm}$ square, as delineated by the slide grids. The pathologist's assessment was based on examination of the slides under a microscope. The histologic slides were used as the gold standard for identifying regions of connective tissue, lipid-rich necrotic core, and hemorrhage. Because specimens were decalcified during fixation before histologic sectioning, the micro-CT images served as the gold standard for identifying calcified regions. The pathologist outlined and labeled the regions corresponding to these 4 components (connective tissue, lipid-rich necrotic core, hemorrhage, and calcifications) on the corresponding digitized images. The tissue classes were defined before classification as follows: regions of collagen strands and elastic fibers, connective tissue matrix, and proteoglycans were termed connective tissue; regions containing cellular debris, a disorganized mass of lipid material and/or cholesterol crystals, cholesterol clefts, and lipidladen foam macrophages were called lipid-rich necrotic core; and regions with blood products or calcifications were called hemorrhage and calcifications, respectively. The areas of each of these regions were computed, and the percentages of connective tissue, with a random effect for patient built interpatient correlation into the calculation of these densities and an assessment of their variability. Because the variances for the different components were similar, the optimal cutoff for differentiating between histologic components was determined to be the halfway Hounsfield attenuation between the mean densities for each of the components.

\section{Qualitative Analysis}

We developed an automated classification computer algorithm that segments the inner (luminal) and outer contours of the carotid artery walls from the in vivo CTA datasets; then, with the Hounsfield attenuation thresholds calculated in the quantitative analysis as described above, the "type" of each image pixel (connective tissue, lipid-rich necrotic core, hemorrhage, and calcifications) located within the carotid wall is assigned. Each of the CTA reformatted images was analyzed by this algorithm, and a color overlay was created affording a visual display of the composition of the carotid wall for each CTA image (Figs 1-4).

A neuroradiologist reviewed these color overlays on the CTA reformatted images (as displayed in Figs 1-4) and characterized the type of atherosclerotic plaque and the stage of lesion development in each quadrant of the carotid wall (0-3 hours, 3-6 hours, 6-9 hours, and 9-12 hours) using a system derived from the American Heart Association (AHA) classification system and adapted for noninvasive image data, such as CT images (Table 1). ${ }^{25,43,44}$ This adaptation was accomplished by combining type I and II lesions, as well as type IV and Va lesions, as proposed previously. ${ }^{45,46}$ Ulceration was defined by the presence of large obvious excavation ( $\geq 2 \mathrm{~mm}$ in depth) on the surface of the plaque, with a well-defined back wall at its base. The maximal thickness of the plaque and the minimal thickness of the 


\begin{tabular}{|c|c|c|}
\hline $\begin{array}{l}\text { Type of } \\
\text { Plaque }\end{array}$ & $\begin{array}{c}\text { AHA Histologic } \\
\text { Classification of } \\
\text { Atherosclerotic Plaques }\end{array}$ & $\begin{array}{c}\text { Classification of } \\
\text { Atherosclerotic Plaques } \\
\text { Adapted for CT }\end{array}$ \\
\hline 0 & No intimal thickening & No intimal thickening \\
\hline 1 & $\begin{array}{l}\text { Initial lesion; not grossly } \\
\text { apparent plaque; foam } \\
\text { cells }\end{array}$ & $\begin{array}{l}\text { Thin plaque with no } \\
\text { calcification }\end{array}$ \\
\hline 2 & $\begin{array}{l}\text { Fatty streak; lesion grossly } \\
\text { apparent; foam cells } \\
\text { and smooth muscle } \\
\text { cells contain lipid } \\
\text { droplets }\end{array}$ & \\
\hline 3 & $\begin{array}{l}\text { Preatheroma; foam cells } \\
\text { contain lipid droplets } \\
\text { and increased number } \\
\text { of smooth muscle cells; } \\
\text { raised fatty streak }\end{array}$ & $\begin{array}{l}\text { Plaque with small lipid cores } \\
\text { and no calcification }\end{array}$ \\
\hline 4 & $\begin{array}{l}\text { Atheroma; massive } \\
\text { extracellular lipid pool } \\
\text { (lipid core); grossly } \\
\text { visible; infiltrated with } \\
\text { foam cells and smooth } \\
\text { muscle cells }\end{array}$ & $\begin{array}{l}\text { Plaque with a large lipid core, } \\
\text { covered by a fibrous cap, } \\
\text { possible small } \\
\text { calcifications }\end{array}$ \\
\hline $5 a$ & $\begin{array}{c}\text { Fibroatheroma; type IV } \\
\text { with a fibrous cap }\end{array}$ & \\
\hline $5 b$ & $\begin{array}{l}\text { Calcified plaque; lesion } \\
\text { with a lipid core or } \\
\text { fibrotic tissue, with } \\
\text { large calcifications }\end{array}$ & $\begin{array}{l}\text { Plaque with a lipid core or } \\
\text { fibrotic tissue, with large } \\
\text { calcifications }\end{array}$ \\
\hline $5 c$ & $\begin{array}{l}\text { Fibrotic plaque: fibrous } \\
\text { connective tissue, no } \\
\text { lipid core }\end{array}$ & $\begin{array}{l}\text { Plaque with fibrous tissue, no } \\
\text { lipid core, possible small } \\
\text { calcifications }\end{array}$ \\
\hline $6 a$ & Ulcerations & Ulcerations \\
\hline $6 b$ & Wide hemorrhage & Wide hemorrhage \\
\hline $6 c$ & Thrombosis & Thrombosis \\
\hline
\end{tabular}

fibrous cap, as automatically calculated by the computer algorithm for each quadrant of each CTA image, were recorded for the CTA images that had a corresponding histologic section. Of note, the fibrous cap thickness measured by the software is the radial distance to the most superficial core of nonconnective tissue (lipid, blood, or calcium). If no lipid core, hemorrhage, or calcium is present in the carotid wall, then the software reports the fibrous cap thickness as being equal to the wall thickness.

Histologic sections were reviewed independently of the CTA reformatted images, according to a similar protocol. The type of atherosclerotic plaque according to the AHA classification system (Table 1), ${ }^{43,44}$ maximal thickness of the plaque, and thickness of the fibrous cap were also assessed and recorded.

Qualitative characterization of carotid plaques relying on CTA and histologic examination were compared with paired $t$ tests for continuous variables and $\mathrm{McNemar}$ tests and unweighted $\kappa$ values for categoric variables.

\section{Results}

\section{Patients and Specimens}

Our study population consisted of 8 patients (all men; mean age, 61 years; age range, 55-69 years) and 8 resulting endar-
Table 2: Mean in-vivo CT Hounsfield density, SD, and $95 \%$ confidence interval for each histologic component*

\begin{tabular}{lccrr}
\hline $\begin{array}{l}\text { Histologic } \\
\text { Component }\end{array}$ & $\begin{array}{c}\text { Mean } \\
\text { (HU) }\end{array}$ & $\begin{array}{c}\text { SD } \\
\text { (HU) }\end{array}$ & \multicolumn{2}{c}{$\begin{array}{c}\text { 95\% Confidence } \\
\text { Interval (HU) }\end{array}$} \\
\hline Lipid-rich necrotic core & 32.6 & 20.0 & -7.4 & 72.5 \\
Connective tissue & 46.4 & 19.9 & 6.6 & 86.2 \\
Hemorrhage & 97.5 & 22.0 & 53.5 & 141.6 \\
Calcifications & 256.7 & 30.2 & 216.3 & 297.1 \\
\hline
\end{tabular}

Note:- $-\mathrm{HU}$ indicates Hounsfield units

* Determined by comparison of each $2 \times 2$-mm square electronically drawn on the in vivo CT reformatted images with corresponding histologic and micro-CT squares.

terectomy specimens. All specimens had lipid and calcium cores on histologic examination in their plaques; a recent intraplaque hemorrhage was present in 1 patient.

There were 9 histologic sections obtained from each specimen, depending on the length of the specimen. Overall, a total of 53 histologic cross-sections were available for review, with 53 matching in vivo CTA and 53 ex vivo micro-CT reformatted images. A total of 733 squares $(2 \times 2 \mathrm{~mm})$ and 212 quadrants were considered for quantitative and qualitative analyses, respectively.

In vivo CTA was obtained 1 to 5 days before the carotid endarterectomy (median, 1.5 days; interquartile range, 1-2.5 days). Time from endarterectomy to micro-CT and initial histologic preparation was kept to a minimum; typically, surgery was performed early in the morning, micro-CT obtained in the afternoon, and histologic fixation done at the end of the day of the surgery.

\section{Quantitative Analysis}

The mean CT Hounsfield attenuation was measured for each of the $2 \times 2$-mm squares that were electronically drawn on the CT reformatted images and considered in the linear regression model with respect to the percentages of connective tissue, lipid-rich necrotic core, hemorrhage, and calcifications in the corresponding histologic and micro-CT squares. The results of the linear mixed model (ie, mean Hounsfield attenuation for each histologic component and the $95 \%$ confidence intervals for these densities) are displayed in Table 2.

There was significant overlap in CT Hounsfield densities between lipid-rich necrotic core and connective tissue. There was also some overlap between connective tissue and hemorrhage. Cutoff densities between lipid-rich necrotic core and connective tissue, connective tissue and hemorrhage, and hemorrhage and calcifications were determined as the halfway Hounsfield attenuation between the average densities for each of the components: 39.5 Hounsfield units (HU) between lipid-rich necrotic core and connective tissue, 72.0 HU between connective tissue and hemorrhage, and 177.1 HU between hemorrhage and calcifications.

\section{Qualitative Analysis}

The comparison of CT classification and gold standard histologic classification of type of atherosclerotic plaque and stage of lesion development according to the system derived from the AHA classification system ${ }^{25,43,44}$ is displayed in Table 3. There was an overall $72.6 \%$ agreement between CTA and histologic examination, corresponding to an unweighted $\kappa$ of 67.6\%. $(P<.001)$ (Figs 1 and 2$)$. For large calcifications (Vb plaques), CTA classified the lesion in perfect concordance with histologic features (Table 4). 
Table 3: Comparison of CTA classification and gold standard histologic classification of type of atherosclerotic plaque and stage of lesion development according to the system derived from the American Heart Association (AHA) classification system

\begin{tabular}{|c|c|c|c|c|c|c|c|c|c|c|c|}
\hline \multicolumn{12}{|c|}{ Histologic Classification } \\
\hline & Type of Plaque & 0 & I-II & III & IV-Va & Vb & Vc & Vla & Vlb & VIc & Total \\
\hline \multirow{10}{*}{ CTA Classification } & 0 & 12 & 2 & & & & & & & & 14 \\
\hline & I-II & 4 & 16 & 8 & & & & & & & 28 \\
\hline & III & & 8 & 35 & 9 & & 6 & 1 & & & 59 \\
\hline & IV-Va & & & & 29 & & & & & & 29 \\
\hline & Vb & & & & & 26 & & & & & 26 \\
\hline & Vc & & & 9 & 2 & & 15 & 1 & 5 & & 32 \\
\hline & Vla & & & & 1 & & 1 & 13 & & & 15 \\
\hline & Vlb & & & & & & 1 & & 8 & & 9 \\
\hline & Vlc & & & & & & & & & & 0 \\
\hline & Total & 16 & 26 & 52 & 41 & 26 & 23 & 15 & 13 & 0 & 212 \\
\hline
\end{tabular}

Note:-CTA indicates CT angiography

Table 4: Comparison of CTA and gold standard histologic examination for large calcifications (Vb plaques)

\begin{tabular}{llrr} 
& & \multicolumn{2}{c}{ Histologic } \\
Large Calcifications & & Examination \\
\cline { 3 - 4 } (Vb Lesions) & No & 186 & Yes \\
\hline CTA & Yes & 0 & 0 \\
& & & 26 \\
\hline
\end{tabular}

Note:-CTA indicates CT angiography.

Table 5: Comparison of CTA and gold standard histologic examination for small and large lipid cores (III, IV-Va,Vb plaques)

\begin{tabular}{llrr} 
& & \multicolumn{2}{c}{$\begin{array}{c}\text { Histologic } \\
\text { Examination }\end{array}$} \\
\cline { 3 - 4 } Small and Large Lipid Cores & & No & Yes \\
(III, IV-Va, Vb Lesions) & No & 69 & 29 \\
\hline CTA & Yes & 24 & 90 \\
\hline
\end{tabular}

Note:-CTA indicates CT angiography.

Table 6: Comparison of CTA and gold standard histologic examination for large lipid cores only (IV-Va plaques)

\begin{tabular}{llrr} 
& & \multicolumn{2}{c}{$\begin{array}{c}\text { Histologic } \\
\text { Examination }\end{array}$} \\
\cline { 3 - 4 } Large Lipid Cores & & No & Yes \\
(IV-Va Lesions) & No & 171 & 12 \\
\hline CTA & Yes & 0 & 29 \\
\hline
\end{tabular}

Note:-CTA indicates CT angiography.

CTA did not perform well in classifying all lipid cores $(\kappa=$ $0.495 ; P=.492$ ) (Table 5 ), likely because the overlap in Hounsfield densities for connective tissues and lipids makes it difficult to distinguish small lipid cores. However, when only large lipid cores ( $\geq 5$ pixels, IV-Va plaques) are considered, CTA classified lesions in greater concordance compared with histologic examination $(\kappa=0.796 ; P<.001)$ (Table 6$)$. The 5 pixels cutpoint was determined by a sensitivity analysis showing that, when 5 pixels or more fall into a class of $\mathrm{HU}$, the specificity in characterizing correctly the corresponding histologic component was superior to $90 \%$.

CTA had similar difficulty in classifying all hemorrhages but again improved when only large hemorrhages ( $\geq 5$ pixels, VIb plaques) were considered $(\kappa=0.712 ; P=.102)$ (Table 7$)$.

CTA also showed strong concordance with histologic features when classifying ulcerations (VIa plaques), which resulted in a $\kappa$ of 0.855 (Table 8 ).

$\begin{aligned} & \text { Table 7: Comparison of CTA and gold standard histologic } \\
& \text { examination for wide hemorrhages (VIb plaques) }\end{aligned}$
\begin{tabular}{llrc} 
& \multicolumn{3}{c}{$\begin{array}{c}\text { Histologic } \\
\text { Examination }\end{array}$} \\
\cline { 2 - 4 } $\begin{array}{l}\text { Wide Hemorrhages } \\
\text { (VIb Plaques) }\end{array}$ & No & Yes \\
\hline CTA & No & 186 & 5 \\
& Yes & 1 & 8
\end{tabular}

Note:-CTA indicates CT angiography.

Table 8: Comparison of CTA and gold standard histologic examination for ulcerations (Vla plaques)

\begin{tabular}{llrr}
\hline \multirow{2}{*}{$\begin{array}{l}\text { Ulcerations } \\
\text { (Vla Plaques) }\end{array}$} & \multicolumn{2}{c}{ Histologic Examination } \\
\cline { 3 - 4 } & & No & Yes \\
\hline CTA & No & 171 & 2 \\
& Yes & 2 & 13 \\
\hline
\end{tabular}

Note:-CTA indicates CT angiography.

Patients with occluded vessels do not typically proceed to endarterectomy, and there was no thrombosed plaque (VIc) among the 8 subjects in our study group.

The mean of the minimal fibrous cap thickness was $0.9 \pm 1.1$ $\mathrm{mm}$ (range, $0.0-5.6 \mathrm{~mm}$ ) on histologic examination, and $1.1 \pm$ $1.0 \mathrm{~mm}$ (range, $0.0-6.6 \mathrm{~mm}$ ) on CTA. Linear regression between CTA and histologic examination in fibrous cap thickness was excellent $(P<.001)$, with a slope of 0.86 (95\% confidence interval, $0.77-0.96)$, an intercept of $0.3 \mathrm{~mm}$ (95\% confidence interval, $0.2-0.5)$ and a coefficient of correlation $R^{2}=0.77$.

The mean of the maximal carotid wall thickness was $3.4 \pm$ $2.4 \mathrm{~mm}$ (range, $0.1-10.5 \mathrm{~mm}$ ) on histologic examination, and $5.1 \pm 2.8 \mathrm{~mm}$ (range, $0.4-11.6 \mathrm{~mm}$ ) on CTA. Linear regression between CTA and histologic examination in carotid wall thickness was significant $(P<.001)$, with a slope of $0.98(95 \%$ confidence interval, $0.86-1.11)$, an intercept of $1.8 \mathrm{~mm}(95 \%$ confidence interval, 1.3-2.3) and a coefficient of correlation $R^{2}=0.72$.

Overestimation of the carotid wall thickness by CTA compared with histologic examination likely resulted from the carotid endarterectomy specimen including only the intima and part of the media, whereas CTA imaging considered the entire carotid wall. The same reason may explain why some plaques that were classified as type 0 or 1 to 2 on histologic examination were classified as 1 to 2 or 3 by CTA. A similar issue was previously reported for MR imaging of the carotid plaques. ${ }^{29}$ 


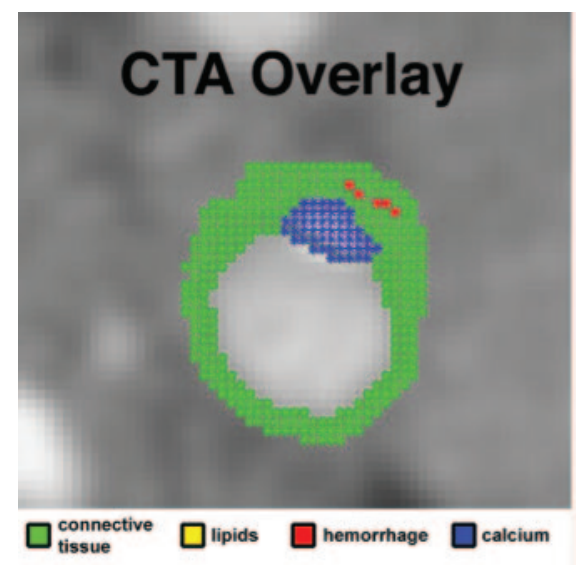

\section{Histology}

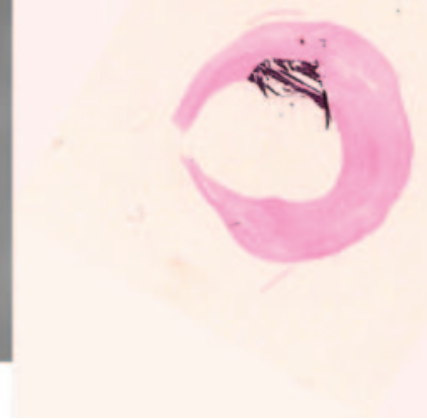

\section{Histology}

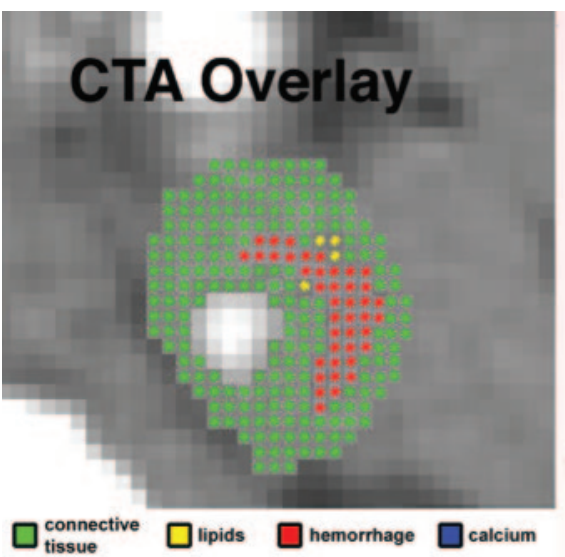

An alternative explanation would be some degree of shrinkage during the histologic preparation.

\section{Discussion}

This study provides proof of principle that the composition of atherosclerotic plaques determined by CTA accurately reflects composition of the lesion as defined by histologic examination. To the best of our knowledge, this is the first report on the use of a CTA-derived, automated classification computer algorithm to distinguish the "type" of each image pixel (connective tissue, lipid-rich necrotic core, hemorrhage, and calcifications) located within the carotid wall. We found that the CTderived algorithm was excellent in classifying calcifications. CTA classification worked less well for classifying lipid-rich necrotic cores and hemorrhage, likely because the range of densities associated with these components overlaps with the densities associated with connective tissue. This overlap severely limited the reliability of individual pixel Hounsfield readings to indicate fibrous tissue, hemorrhage, or lipid-rich necrotic cores; it posed less of a limitation when larger areas of lesion were considered. Indeed, CTA classification showed good correlation with histologic examination when only large lipid cores and large hemorrhages were considered. CTA classification also performed well in detecting ulcerations and in measuring the fibrous cap thickness.

The results of our study are in agreement with previous studies that used CT to characterize atherosclerotic plaque in carotid, $^{31,32,35-37,40}$ popliteal, $^{39}$ or coronary ${ }^{38,39,41}$ arteries. This was most notable with regard to identifying calcifica-
Fig 3. In vivo CTA image of the ICA, and matching ex vivo histologic section. Automated classification computer algorithm-derived overlay demonstrates a plaque with a superficial calcification (blue), making it a Vc lesion according to the AHA classification, in agreement with histologic examination.
Fig 4. In vivo CTA image of the ICA, and matching ex vivo histologic section. Automated classification computer algorithm-derived overlay demonstrates a plaque with a large hemorrhage (red), making it a Vlb lesion according to the AHA classification, in agreement with histologic examination. tions, ${ }^{36-39}$ and concerning identifying attenuation ranges for the different plaque components. ${ }^{31,32,40}$ Our study also supports previous findings that CT can play a role in the identification of carotid plaque ulcerations. ${ }^{30}$ In contrast to our study, previous studies did not use a systematic approach to identify all components of carotid atherosclerotic plaque but either adopted a qualitative approach consisting of drawing regions of interest around supposed components, ${ }^{31,39,40}$ or simply averaged the attenuation for the entire arterial wall seen on a section, without stereologic differentiation. ${ }^{35,37}$ Previous studies typically focused on only 1 specific plaque component such as calcium, differentiating between calcified and noncalcified plaques, without going further in the characterization of the components. ${ }^{32,36,38,39,41}$

Our study is unique in several ways. We used modern multidetector-row CT scanners with rapid acquisition, no motion artifact, and better contrast profiles and enhancement, whereas previous studies have reported on older generations of CT scanners (some single-section) $)^{31,32,35-40}$ with relatively thick $\left(3 \mathrm{~mm}\right.$ ) sections. ${ }^{35,37}$ In our study patients, we optimized the intraluminal enhancement using a bolus timing strategy to time our CTA acquisition to the early arterial phase. The high intraluminal enhancement that was obtained with this approach increased the accuracy of the segmentation of the inner contour of the carotid artery wall by the software, by reinforcing the contrast between the lumen and the wall. We used an automated, computed analysis rather than an observer interpretation as in previous studies. ${ }^{29}$ Automated classification algorithms have previously been proposed with MR imag- 
ing $^{25,27,47,48}$ but not CT. Automated classification algorithms, such as the one presented in our study, could lead to improved reproducibility in characterization of plaques and could be of interest in longitudinal studies of progression of atherosclerotic disease. ${ }^{24,49}$

Unlike sonography, CT cannot be performed at the bedside, and it has inferior tissue contrast resolution compared with MR imaging. However, CTA of the carotid arteries is a routine imaging test that is frequently obtained as part of the standard of care of patients with acute or chronic cerebrovascular disease. At the present time, interpretation of CTA studies focuses on the degree of luminal narrowing. Our study shows that attention should also be paid to the characteristics of the carotid wall as demonstrated on CTA, because they reflect histologic composition. CTA provides an absolute quantitative measure of tissue composition, whereas signal intensity on MR imaging is only a relative value. This information is included in the dataset obtained as part of the standard-ofcare CTA. The potential clinical applications of our results are enhanced by the fact that CTA can be obtained in a few seconds in the clinical setting and does not require any specific research imaging protocol.

Our study had several limitations. We did not correlate the plaque composition with patients' symptoms because of the limited sample size and that all 8 patients presented with TIAs. During the quantitative analysis, we did not perform a pixelby-pixel comparison but, rather, a small square-by-smallsquare comparison. The resulting heterogeneity of tissue within squares was associated with a corresponding heterogeneity of attenuations and was taken into account by the linear mixed model analysis. After quantitative analysis, we did not calculate the sensitivity, specificity, negative predictive value, positive predictive value, or accuracy of CT for determining the different components. Rather, we evaluated whether the thresholds afforded could characterize the types of plaques, which are more clinically relevant. We did not examine the degree of luminal narrowing on CTA in our study participants because this was examined in a different study.

Finally, because our algorithm and the characterization of the plaques were validated in the same sample in which the thresholds were defined, there was the potential for an overfitting bias. The strong agreement between CTA and histologic classification of plaques could be overly optimistic given this limitation. To address this issue, validation of the algorithm in a different, prospective sample of patients is required.

In conclusion, the composition of carotid atherosclerotic plaques determined by CTA accurately reflects the composition of plaques defined on histologic examination. The ability to analyze components of carotid plaques on CTA with an automated classification algorithm could provide a convenient, repeatable, noninvasive method of studying carotid atherosclerotic disease in longitudinal studies. Correlation of CTA-derived assessment of carotid plaques with symptoms and risk for stroke remains to be investigated.

\section{Acknowledgments}

We thank Bernard P. Halloran, PhD, from the Division of Endocrinology, Veterans Affairs Medical Center, University of
California, San Francisco, for generously granting us access to his micro-CT scanner.

\section{References}

1. Beneficial effect of carotid endarterectomy in symptomatic patients with high-grade carotid stenosis. North American Symptomatic Carotid Endarterectomy Trial Collaborators. N Engl J Med 1991;325:445-53

2. Barnett HJ, Taylor DW, Eliasziw M, et al. Benefit of carotid endarterectomy in patients with symptomatic moderate or severe stenosis. North American Symptomatic Carotid Endarterectomy Trial Collaborators. N Engl J Med 1998;339:1415-25

3. Randomised trial of endarterectomy for recently symptomatic carotid stenosis: final results of the MRC European Carotid Surgery Trial (ECST). Lancet 1998;351:1379-87

4. Mayberg MR, Wilson SE, Yatsu F, et al. Carotid endarterectomy and prevention of cerebral ischemia in symptomatic carotid stenosis. Veterans Affairs Cooperative Studies Program 309 Trialist Group. JAMA 1991;266:3289-94

5. Ebrahim S, Papacosta O, Whincup P, et al. Carotid plaque, intima media thickness, cardiovascular risk factors, and prevalent cardiovascular disease in men and women: the British Regional Heart Study. Stroke 1999;30:841-50

6. O'Leary DH, Polak JF, Kronmal RA, et al. Distribution and correlates of sonographically detected carotid artery disease in the Cardiovascular Health Study. The CHS Collaborative Research Group. Stroke 1992;23:1752-60

7. Glagov S, Weisenberg E, Zarins CK, et al. Compensatory enlargement of human atherosclerotic coronary arteries. $N$ Engl J Med 1987;316:1371-75

8. Ballotta E, Da Giau G, Renon L. Carotid plaque gross morphology and clinical presentation: a prospective study of 457 carotid artery specimens. J Surg Res 2000;89:78-84

9. Lovett JK, Gallagher PJ, Hands LJ, et al. Histological correlates of carotid plaque surface morphology on lumen contrast imaging. Circulation 2004;110:2190-97

10. McCarthy MJ, Loftus IM, Thompson MM, et al. Angiogenesis and the atherosclerotic carotid plaque: an association between symptomatology and plaque morphology. J Vasc Surg 1999;30:261-68

11. Naghavi M, Libby P, Falk E, et al. From vulnerable plaque to vulnerable patient: a call for new definitions and risk assessment strategies: Part I. Circulation 2003;108:1664-72

12. Rothwell PM, Gibson R, Warlow CP. Interrelation between plaque surface morphology and degree of stenosis on carotid angiograms and the risk of ischemic stroke in patients with symptomatic carotid stenosis. On behalf of the European Carotid Surgery Trialists' Collaborative Group. Stroke 2000;31:615-21

13. Bonithon-Kopp C, Scarabin PY, Taquet A, et al. Risk factors for early carotid atherosclerosis in middle-aged French women. Arterioscler Thromb 1991;11:966-72

14. Bonithon-Kopp C, Touboul PJ, Berr C, et al. Relation of intima-media thickness to atherosclerotic plaques in carotid arteries. The Vascular Aging (EVA) Study. Arterioscler Thromb Vasc Biol 1996;16:310-16

15. Lorenz MW, von Kegler S, Steinmetz H, et al. Carotid intima-media thickening indicates a higher vascular risk across a wide age range: prospective data from the Carotid Atherosclerosis Progression Study (CAPS). Stroke 2006;37:87-92

16. O'Leary DH, Polak JF, Kronmal RA, et al. Carotid-artery intima and media thickness as a risk factor for myocardial infarction and stroke in older adults. Cardiovascular Health Study Collaborative Research Group. N Engl J Med 1999;340:14-22

17. Zureik M, Touboul PJ, Bonithon-Kopp C, et al. Cross-sectional and 4-year longitudinal associations between brachial pulse pressure and common carotid intima-media thickness in a general population. The EVA study. Stroke 1999;30:550-55

18. Bassiouny HS, Sakaguchi Y, Mikucki SA, et al. Juxtalumenal location of plaque necrosis and neoformation in symptomatic carotid stenosis. J Vasc Surg 1997;26:585-94

19. Biasi GM, Froio A, Diethrich EB, et al. Carotid plaque echolucency increases the risk of stroke in carotid stenting: the Imaging in Carotid Angioplasty and Risk of Stroke (ICAROS) study. Circulation 2004;110:756-62

20. Prabhakaran S, Rundek T, Ramas R, et al. Carotid plaque surface irregularity predicts ischemic stroke: the northern Manhattan study. Stroke 2006;37:2696-701

21. Miralles M, Merino J, Busto M, et al. Quantification and characterization of carotid calcium with multi-detector CT-angiography. Eur J Vasc Endovasc Surg 2006;32:561-67

22. Carr S, Farb A, Pearce WH, et al. Atherosclerotic plaque rupture in symptomatic carotid artery stenosis. J Vasc Surg 1996;23:755-65; discussion 765-56

23. Glagov S, Bassiouny HS, Giddens DP, et al. Pathobiology of plaque modeling and complication. Surg Clin North Am 1995;75:545-56

24. Adame IM, van der Geest RJ, Wasserman BA, et al. Automatic segmentation and plaque characterization in atherosclerotic carotid artery MR images. MAGMA 2004;16:227-34

25. Clarke SE, Hammond RR, Mitchell JR, et al. Quantitative assessment of carotid 
plaque composition using multicontrast MRI and registered histology. Magn Reson Med 2003;50:1199-208

26. Coombs BD, Rapp JH, Ursell PC, et al. Structure of plaque at carotid bifurcation: high-resolution MRI with histological correlation. Stroke 2001;32:2516-21

27. Shinnar M, Fallon JT, Wehrli S, et al. The diagnostic accuracy of ex vivo MRI for human atherosclerotic plaque characterization. Arterioscler Thromb Vasc Biol 1999;19:2756-61

28. Worthley SG, Helft G, Fuster V, et al. Serial in vivo MRI documents arterial remodeling in experimental atherosclerosis. Circulation 2000;101:586-89

29. Yuan C, Mitsumori LM, Ferguson MS, et al. In vivo accuracy of multispectral magnetic resonance imaging for identifying lipid-rich necrotic cores and intraplaque hemorrhage in advanced human carotid plaques. Circulation 2001;104:2051-56

30. Cinat M, Lane CT, Pham $\mathrm{H}$, et al. Helical CT angiography in the preoperative evaluation of carotid artery stenosis. J Vasc Surg 1998;28:290-300

31. Estes JM, Quist WC, Lo Gerfo FW, et al. Noninvasive characterization of plaque morphology using helical computed tomography. J Cardiovasc Surg (Torino) 1998;39:527-34

32. Oliver TB, Lammie GA, Wright AR, et al. Atherosclerotic plaque at the carotid bifurcation: CT angiographic appearance with histopathologic correlation. AJNR Am J Neuroradiol 1999;20:897-901

33. Anderson GB, Ashforth R, Steinke DE, et al. CT angiography for the detection and characterization of carotid artery bifurcation disease. Stroke 2000;31:2168-74

34. Leclerc X, Godefroy O, Pruvo JP, et al. Computed tomographic angiography for the evaluation of carotid artery stenosis. Stroke 1995;26:1577-81

35. Culebras A, Leeson MD, Cacayorin ED, et al. Computed tomographic evaluation of cervical carotid plaque complications. Stroke 1985;16:425-31

36. Gronholdt ML. B-mode ultrasound and spiral CT for the assessment of carotid atherosclerosis. Neuroimaging Clin N Am 2002;12:421-35

37. Gronholdt ML, Wagner A, Wiebe BM, et al. Spiral computed tomographic imaging related to computerized ultrasonographic images of carotid plaque morphology and histology. J Ultrasound Med 2001;20:451-58

38. Leber AW, Becker A, Knez A, et al. Accuracy of 64-slice computed tomography to classify and quantify plaque volumes in the proximal coronary system: a comparative study using intravascular ultrasound. J Am Coll Cardiol 2006;47:672-77
39. Schroeder S, Kuettner A, Leitritz M, et al. Reliability of differentiating human coronary plaque morphology using contrast-enhanced multislice spiral computed tomography: a comparison with histology. J Comput Assist Tomogr 2004;28:449-54

40. Walker LJ, Ismail A, McMeekin W, et al. Computed tomography angiography for the evaluation of carotid atherosclerotic plaque: correlation with histopathology of endarterectomy specimens. Stroke 2002;33:977-81

41. Becker CR, Nikolaou K, Muders M, et al. Ex vivo coronary atherosclerotic plaque characterization with multi-detector-row CT. Eur Radiol 2003;13:2094-98

42. Lovett JK, Redgrave JN, Rothwell PM. A critical appraisal of the performance, reporting, and interpretation of studies comparing carotid plaque imaging with histology. Stroke 2005;36:1091-97

43. Stary HC, Chandler AB, Dinsmore RE, et al. A definition of advanced types of atherosclerotic lesions and a histological classification of atherosclerosis. A report from the Committee on Vascular Lesions of the Council on Arteriosclerosis, American Heart Association. Circulation 1995;92:1355-74

44. Stary HC, Chandler AB, Glagov S, et al. A definition of initial, fatty streak, and intermediate lesions of atherosclerosis. A report from the Committee on Vascular Lesions of the Council on Arteriosclerosis, American Heart Association. Circulation 1994;89:2462-78

45. Fayad ZA, Nahar T, Fallon JT, et al. In vivo magnetic resonance evaluation of atherosclerotic plaques in the human thoracic aorta: a comparison with transesophageal echocardiography. Circulation 2000;101:2503-09

46. Cai JM, Hatsukami TS, Ferguson MS, et al. Classification of human carotid atherosclerotic lesions with in vivo multicontrast magnetic resonance imaging. Circulation 2002;106:1368-73

47. Clarke SE, Beletsky V, Hammond RR, et al. Validation of automatically classified magnetic resonance images for carotid plaque compositional analysis. Stroke 2006;37:93-97

48. Itskovich VV, Samber DD, Mani V, et al. Quantification of human atherosclerotic plaques using spatially enhanced cluster analysis of multicontrastweighted magnetic resonance images. Magn Reson Med 2004;52:515-23

49. Adams GJ, Greene J, Vick GW 3rd, et al. Tracking regression and progression of atherosclerosis in human carotid arteries using high-resolution magnetic resonance imaging. Magn Reson Imaging 2004;22:1249-58 